\title{
Recalibration and validation of the Charlson Comorbidity Index in acute kidney injury patients underwent continuous renal replacement therapy
}

\author{
Jinwoo Lee ${ }^{1}$, Jiyun Jung ${ }^{2,3}$, Jangwook Lee ${ }^{1,3}$, Jung Tak Park ${ }^{4}$, Chan-Young Jung ${ }^{4}$, Yong Chul Kim ${ }^{5}$, Dong Ki Kim ${ }^{5}$, \\ Jung Pyo Lee ${ }^{6,7}$, Sung Jun Shin ${ }^{1,3,7}$, Jae Yoon Park ${ }^{1,3,7}$ \\ ${ }^{1}$ Department of Internal Medicine, Dongguk University Ilsan Hospital, Goyang, Republic of Korea \\ ${ }^{2}$ Data Management and Statistics Institute, Dongguk University Ilsan Hospital, Goyang, Republic of Korea \\ ${ }^{3}$ Research Center for Chronic Disease and Environmental Medicine, Dongguk University Ilsan Hospital, Goyang, Republic of Korea \\ ${ }^{4}$ Department of Internal Medicine, Yonsei University College of Medicine, Seoul, Republic of Korea \\ ${ }^{5}$ Department of Internal Medicine, Seoul National University College of Medicine, Seoul, Republic of Korea \\ ${ }^{6}$ Department of Internal Medicine, SMG-SNU Boramae Medical Center, Seoul, Republic of Korea \\ ${ }^{7}$ Department of Internal Medicine, Dongguk University College of Medicine, Goyang Republic of Korea
}

Background: Comorbid conditions impact the survival of patients with severe acute kidney injury (AKI) who require continuous renal replacement therapy (CRRT). The weights assigned to comorbidities in predicting survival vary based on type of index, disease, and advances in management of comorbidities. We developed a modified Charlson Comorbidity Index (CCI) for use in patients with AKI requiring CRRT (mCCl-CRRT) and improved the accuracy of risk stratification for mortality.

Methods: A total of 828 patients who received CRRT between 2008 and 2013, from three university hospital cohorts was included to develop the comorbidity score. The weights of the comorbidities were recalibrated using a Cox proportional hazards model adjusted for demographic and clinical information. The modified index was validated in a university hospital cohort $(n=919)$ using the data of patients treated from 2009 to 2015.

Results: Weights for dementia, peptic ulcer disease, any tumor, and metastatic solid tumor were used to recalibrate the mCCI-CRRT. Use of these calibrated weights achieved a 35.4\% (95\% confidence interval [Cl], 22.1\%-48.1\%) higher performance than unadjusted $\mathrm{CCl}$ in reclassification based on continuous net reclassification improvement in logistic regression adjusted for age and sex. After additionally adjusting for hemoglobin and albumin, consistent results were found in risk reclassification, which improved by $35.9 \%$ (95\% $\mathrm{Cl}, 23.3 \%-48.5 \%)$.

Conclusion: The mCCI-CRRT stratifies risk of mortality in AKI patients who require CRRT more accurately than does the original CCI, suggesting that it could serve as a preferred index for use in clinical practice.

Keywords: Acute kidney injury, Charlson Comorbidity Index, Continuous renal replacement therapy, Mortality, Risk assessment

Received: May 14, 2021; Revised: August 31, 2021; Accepted: September 14, 2021

Correspondence: Jae Yoon Park

Department of Internal Medicine, Dongguk University Ilsan Hospital, 27 Dongguk-ro, Ilsandong-gu, Goyang 10326, Republic of Korea. E-mail: nephrojyp@gmail.com

ORCID: https://orcid.org/0000-0001-8986-7492

Jinwoo Lee and Jiyun Jung contributed equally to this work as co-first authors.

Copyright (C) 2022 by The Korean Society of Nephrology

(a) This is an Open Access article distributed under the terms of the Creative Commons Attribution Non-Commercial and No Derivatives License (http:// creativecommons.org/licenses/by-nc-nd/4.0/) which permits unrestricted non-commercial use, distribution of the material without any modifications, and reproduction in any medium, provided the original works properly cited. 


\section{Introduction}

Despite advances in critical care medicine over the past decades, treatment outcomes for patients admitted to the intensive care unit (ICU) with acute kidney injury (AKI) remain poor [1,2]. Since continuous renal replacement therapy (CRRT), developed by Kramer in 1977, has become an indispensable treatment option for critical care, the mortality rate of severe AKI patients who require CRRT has remained very high over the past 40 years, likely due to the increased severity of underlying disease. Since the number and severity of underlying comorbid diseases are among the main causes of such an increase [1-3], predicting prognosis using an appropriate scoring system is important in treating critically ill AKI patients as well as for providing and allocating medical resources.

The Charlson Comorbidity Index (CCI) is the most widely used tool to evaluate the effects of comorbid diseases on patient prognosis, and its use has been validated in various acute/chronic conditions, including kidney diseases [4]. However, since the CCI was created originally as an index for general ward-admitted patients with various comorbid diseases, it is questionable whether each disease covered by the index exerts the same impact in severe AKI patients requiring CRRT. Since the index was developed 30 years ago, there is an inherent disadvantage that the effects of the diseases addressed might have changed with treatment technology. Recently, various tools such as the Davies index and the Index of Coexistent Disease score [5-7] have been created to evaluate the burden of coexistent chronic diseases. Nevertheless, the CCI remains the most widely used and clinician-friendly scoring system [4]. Although a series of studies has recalibrated and validated CCI for patients undergoing peritoneal dialysis, hemodialysis, and renal transplantation, research regarding CRRT patients is lacking [8-10]. Therefore, we recalibrated the weight of comorbidities on mortality in severe AKI patients requiring CRRT by modifying the original CCI (mCCI) and validated the performance of the new mCCI index compared with that of the original CCI.

\section{Methods}

\section{Data collection in the development cohort}

We collected data from 858 AKI patients aged $\geq 18$ years who received CRRT from Seoul National University Hospital (n = 439), Seoul National University Boramae Medical Center $(\mathrm{n}=218)$, or Dongguk University Ilsan Hospital $(\mathrm{n}=201)$ between 2008 and 2013, as the development cohort. We excluded patients who had incomplete data regarding hemoglobin and albumin levels $(\mathrm{n}=30)$. Therefore, the following demographic and clinical information were obtained for 828 AKI patients: sex, age, hemoglobin and albumin at baseline, 28-day mortality, and the 15 comorbidities constituting the CCI (peripheral vascular disease, dementia, myocardial infarction, congestive heart failure, cerebrovascular disease, hemiplegia, diabetes, diabetes with end organ damage, chronic pulmonary disease, connective tissue disease, peptic ulcer disease, any tumor, metastatic solid tumor, mild liver disease, and moderate to severe liver disease), defined based on the International Classification of Disease, the 10th Revision (ICD-10). Although there is a criterion for classifying moderate/severe renal disease in the original CCI appendix, scoring for underlying kidney disease was excluded. This is because our study population was composed of CRRT patients, who are those with severe AKI. These data were confirmed by trained clinicians at each medical center.

This study is in compliance with the Declaration of Helsinki and received full approval from the Institutional Review Boards of Seoul National University Hospital (No. H-1404-028-568), Seoul National University Boramae Medical Center (No. 26-2014-15), Yonsei University Severance Hospital (No. 4-2021-0082), and Dongguk University Ilsan Hospital (No. DUIH 2018-12-010-012). Informed consent was waived for this study because retrospective data were used.

\section{Data collection in the validation cohort}

A total of 1,144 patients who received CRRT at Yonsei University Severance Hospital participated in the validation cohort between 2009 and 2015. We excluded 225 patients with missing information regarding albumin $(\mathrm{n}=216)$ and comorbidities $(n=9)$. Finally, information on age, sex, 
albumin, hemoglobin, and CCI score of 919 patients was collected.

\section{Statistical analyses}

Baseline characteristics in the development and validation cohorts were described using mean and standard deviation for continuous variables and frequency and percentile for categorical variables. The primary outcome of this study was 28-day all-cause mortality, which was observed in each hospitalization. To estimate the calibrated weights of 15 comorbidities in AKI patients, Cox proportional hazard models were used after being stratified by age $(<50$, $50-59$, and $\geq 60$ years) and treatment center in the development cohort and adjusted for sex, albumin, hemoglobin, and the 15 comorbidities. We tested the proportional hazard assumption using the Schoenfeld test. After estimating the hazard ratios (HRs) of the comorbidities in the survival model, the modified CCI of CRRT patients (mCCI-CRRT) was calculated using each significant HR among the CCI diseases divided by the lowest significant HR. The comorbidity score of the mCCI-CRRT for each patient was the sum of the rounded weights. We obtained Kaplan-Meier curves stratified into three categories $(<2,2-4$, $\geq 5$ ) for the original CCI and the mCCI-CRRT scores in the development and validation cohorts to compare the performances. The index discriminations between CCI and mCCI-CRRT were assessed by C-statistics and continuous net reclassification improvement (cNRI). The C-statistic was the area under the receiver operator curve between sensitivity and 1-specificity. To overcome the disadvantages of C-statistics, such as difficulty in clinical interpretation and unclear decision threshold, cNRI, which is the sum of the proportion in event prediction of the event group ( $\left.\mathrm{cNRI}_{\text {event }}\right)$ and the non-event prediction of the nonevent group (cNRI non-event ) was used [11]. Improvement in the mortality prediction of mCCI-CRRT compared to the age-adjusted and original CCI was estimated using a logistic regression model adjusted for sex and age in model 1 and additionally adjusted for hemoglobin and albumin in model 2. All analyses were conducted using R software, version 4.0.2 (R Foundation for Statistical Computing, Vienna, Austria) and $\mathrm{p}<0.05$ was considered statistically significant.

\section{Results}

Baseline characteristics of both the development and validation cohorts are presented in Table 1 . In the development cohort, most participants were in the $\geq 60$-year age group at initiation of CRRT therapy, and $61.0 \%$ of patients were male. Similar distributions were observed regarding age and sex in the validation cohort. The average follow-up duration and 28-day mortality rate during hospitalization were 14.45 \pm 11.96 days and $61.5 \%(n=509)$ in the development cohort, respectively, and $18.92 \pm 11.25$ days and $58.8 \%(n=540)$ in the validation cohort. In the development cohort, most subjects $(82.6 \%)$ had one or more comorbidities. Among the 15 comorbidities, tumor was the most prevalent (24.4\%), followed by diabetes without end organ damage (19.6\%), moderate to severe liver disease (18.1\%), and congestive heart failure (18.0\%). Additionally, $34.5 \%$ of the patients in the validation cohort had diabetes without end organ damage, followed by any tumor (31.9\%), diabetes with end organ damage (24.4\%), and congestive heart disease (16.5\%).

Table 2 lists the $\beta$ coefficients, adjusted HR, and weights for each comorbidity in the Cox proportional hazard model. We confirmed a relationship between time and residuals in proportional hazard assumption using the Schoenfeld test $(\mathrm{p}=0.76)$. The original CCI calculated the adjusted relative risk of each item for 1-year mortality. A relative risk of $<1.2$ was excluded from the weight calculation. Relative risk from 1.2 to $<1.5$ was set as a weight of 1 , from 1.5 to $<2.5$ as a weight of 2 , from 2.5 to $<3.5$ as a weight of 3 , and relative risk $\geq 3.5$ was set as a weight of 6 [4]. Unlike the original CCI, weighting of the mCCI-CRRT was derived by dividing the significant HR of each item by the significant smallest adjusted HR (peptic ulcer disease). Metastatic solid tumor scored 5 points and was the strongest predictor of mortality among the comorbidities, followed by a score of 3 for any tumor (including leukemia and lymphoma) and a score of 1 for dementia and peptic ulcer disease.

The total scores of the original and recalibrated weights were allocated to each patient in the development cohort. The median CCI and mCCI-CRRT in the development cohort were 2 and 0 , respectively (Fig. 1). We categorized the CCI and mCCI-CRRT into three score categories using the same cutoff values $(<2,2-4, \geq 5)$ to compare the probability of survival using CCI and mCCI-CRRT. Fig. 2 illustrates the Kaplan-Meier curves for the CCI (Fig. 2A, C) and mC- 
Table 1. Descriptive characteristics in the development and validation cohorts

\begin{tabular}{|c|c|c|}
\hline Variable & Development cohort & Validation cohort \\
\hline No. of subjects & 828 & 919 \\
\hline \multicolumn{3}{|l|}{ Age $(y r)$} \\
\hline$<50$ & $111(13.4)$ & $162(17.6)$ \\
\hline $50-59$ & $132(15.9)$ & $172(18.7)$ \\
\hline$\geq 60$ & $585(70.7)$ & $585(63.7)$ \\
\hline \multicolumn{3}{|l|}{ Sex } \\
\hline Male & $505(61.0)$ & $567(61.7)$ \\
\hline Female & $323(39.0)$ & $352(38.3)$ \\
\hline 28-Day mortality & $509(61.5)$ & $540(58.8)$ \\
\hline Follow-up duration (day) & $14.45 \pm 11.96$ & $18.92 \pm 11.25$ \\
\hline Hemoglobin (g/dL) & $9.86 \pm 2.25$ & $9.66 \pm 2.26$ \\
\hline Albumin (g/dL) & $2.68 \pm 0.55$ & $2.71 \pm 0.52$ \\
\hline \multicolumn{3}{|l|}{ Comorbidity } \\
\hline No comorbidity & $144(17.4)$ & $140(15.2)$ \\
\hline Peripheral vascular disease & $23(2.8)$ & $36(3.9)$ \\
\hline Dementia & $32(3.9)$ & $33(3.6)$ \\
\hline Myocardial infarction & $98(11.8)$ & $86(9.4)$ \\
\hline Congestive heart failure & $149(18.0)$ & $152(16.5)$ \\
\hline Cerebrovascular disease & $74(8.9)$ & $91(9.9)$ \\
\hline Hemiplegia & $44(5.3)$ & $20(2.2)$ \\
\hline Diabetes, without end organ damage & $162(19.6)$ & $317(34.5)$ \\
\hline Diabetes, with end organ damage & $134(16.2)$ & $224(24.4)$ \\
\hline Chronic pulmonary disease & $31(3.7)$ & $66(7.2)$ \\
\hline Connective tissue disease & $26(3.1)$ & $22(2.4)$ \\
\hline Peptic ulcer disease & $49(5.9)$ & $105(11.4)$ \\
\hline Any tumor & $202(24.4)$ & $293(31.9)$ \\
\hline Metastatic solid tumor & $25(3.0)$ & $89(9.7)$ \\
\hline Mild liver disease & $69(8.3)$ & $119(12.9)$ \\
\hline Moderate to severe liver disease & $150(18.1)$ & $76(8.3)$ \\
\hline Charlson Comorbidity Index score & $3.4 \pm 2.35$ & $3.16 \pm 2.28$ \\
\hline APACHE II score & $28.16 \pm 7.69$ & $27.47 \pm 7.65$ \\
\hline SOFA score & $12.83 \pm 3.41$ & $13.33 \pm 3.59$ \\
\hline
\end{tabular}

Data are expressed as number only, number (\%), or mean \pm standard deviation.

APACHE II, Acute Physiology And Chronic Health Evaluation II; SOFA, sequential organ failure assessment.

CI-CRRT (Fig. 2B, D) differentiated by the three risk groups in both cohorts. When the survival probability was classified by CCI in the development (Fig. 2A) and validation (Fig. 2C) cohorts, survival curves for the $\geq 5$ and 2 to 4 groups intersected and overlapped, while group differences based on the log-rank test were significant in the validation cohort. However, survival curves of mCCI-CRRT (Fig. 2B) showed differences in survival probability according to the classification of risk scores in the development cohort $(\mathrm{p}<0.01)$. In addition, survival probability analysis showed a decrease in mCCI-CRRT (Fig. 2D) as risk increased, with significant between-group differences in the validation cohort.

We estimated the mortality prediction of mCCI-CRRT compared to those of CCI and age-adjusted CCI in the validation cohort using C-statistics (Table 3). Significant difference was observed in the comparison between CCI (0.54; 95\% confidence interval [CI], 0.50-0.58) and mCCI-CRRT (0.59; 95\% CI, 0.55-0.62) ( $\mathrm{p}=0.01)$. However, the C-statistics difference in the age-adjusted CCI (0.58; 95\% CI, 0.54-0.61) compared with that of the mCCI-CRRT was not significant ( $\mathrm{p}$ $=0.60$ ). Our calibrated weights in the mCCI-CRRT achieved a $35.4 \%$ (95\% CI, $22.1 \%-48.1 \%$ ) higher performance than 
Table 2. Weights of mCCI-CRRT based on Cox proportional hazard model compared with original CCI

\begin{tabular}{|c|c|c|c|c|c|c|}
\hline Variable & Coef & SE & $\mathrm{HR}$ & $p$-value & $\mathrm{CCl}$ & mCCl-CRRT \\
\hline Female sex & 0.07 & 0.09 & 1.08 & 0.43 & & \\
\hline Albumin & -0.44 & 0.09 & 0.65 & 0.001 & & \\
\hline Hemoglobin & 0.03 & 0.02 & 1.03 & 0.22 & & \\
\hline Peripheral vascular disease & -0.14 & 0.29 & 0.87 & 0.62 & 1 & 0 \\
\hline Dementia & -0.58 & 0.27 & 0.56 & 0.03 & 1 & 1 \\
\hline Myocardial infarction & -0.06 & 0.17 & 0.94 & 0.72 & 1 & 0 \\
\hline Congestive heart failure & -0.16 & 0.15 & 0.85 & 0.29 & 1 & 0 \\
\hline Cerebrovascular disease & -0.17 & 0.16 & 0.84 & 0.28 & 1 & 0 \\
\hline Hemiplegia & -0.46 & 0.24 & 0.63 & 0.06 & 2 & 0 \\
\hline Diabetes, without end organ damage & -0.12 & 0.12 & 0.89 & 0.31 & 1 & 0 \\
\hline Diabetes, with end organ damage & -0.19 & 0.13 & 0.83 & 0.17 & 2 & 0 \\
\hline Chronic pulmonary disease & 0.26 & 0.2 & 1.30 & 0.20 & 1 & 0 \\
\hline Connective tissue disease & 0.14 & 0.25 & 1.15 & 0.57 & 1 & 0 \\
\hline Peptic ulcer disease & -0.68 & 0.22 & 0.51 & 0.001 & 1 & 1 \\
\hline Any tumor & 0.40 & 0.11 & 1.50 & 0.001 & 2 & 3 \\
\hline Metastatic solid tumor & 0.87 & 0.24 & 2.38 & 0.001 & 6 & 5 \\
\hline Mild liver disease & 0.00 & 0.17 & 1.00 & 0.99 & 1 & 0 \\
\hline Moderate to severe liver disease & 0.14 & 0.12 & 1.15 & 0.24 & 3 & 0 \\
\hline
\end{tabular}

$\mathrm{CCl}$, Charlson Comorbidity Index; Coef, coefficient; HR, hazard ratio; mCCl-CRRT, modified CCI-continuous renal replacement therapy; SE, standard error.
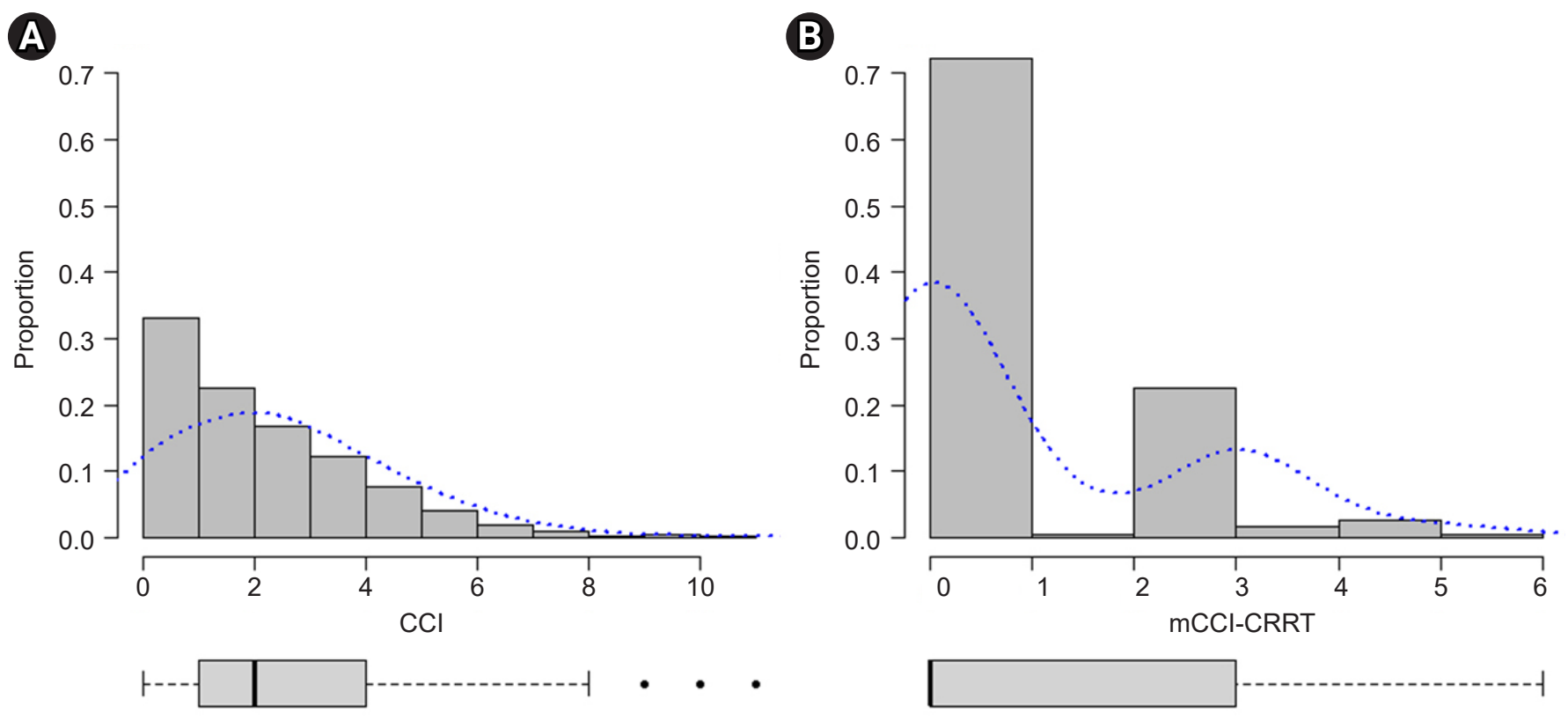

Figure 1. Distribution of the $\mathrm{CCl}(\mathrm{A})$ and $\mathrm{mCCl}-\mathrm{CRRT}(\mathrm{B})$ in the development cohort.

$\mathrm{CCl}$, Charlson Comorbidity Index; mCCl-CRRT, modified CCl-continuous renal replacement therapy.

did CCI in reclassification, based on cNRI in logistic regression adjusted for age and sex. After additionally adjusting for hemoglobin and albumin, consistent results were found in risk reclassification improvement by $35.9 \%$ (95\% CI,
23.3\%-48.5\%). In comparison with age-adjusted CCI, mCCI-CRRT significantly improved the net risk reclassification of mortality by $22.9 \%$ (95\% CI, 9.9\%-35.8\%) in model 1 and by $15.2 \%$ (95\% CI, $2.1 \%-28.2 \%$ ) in model 2 . 

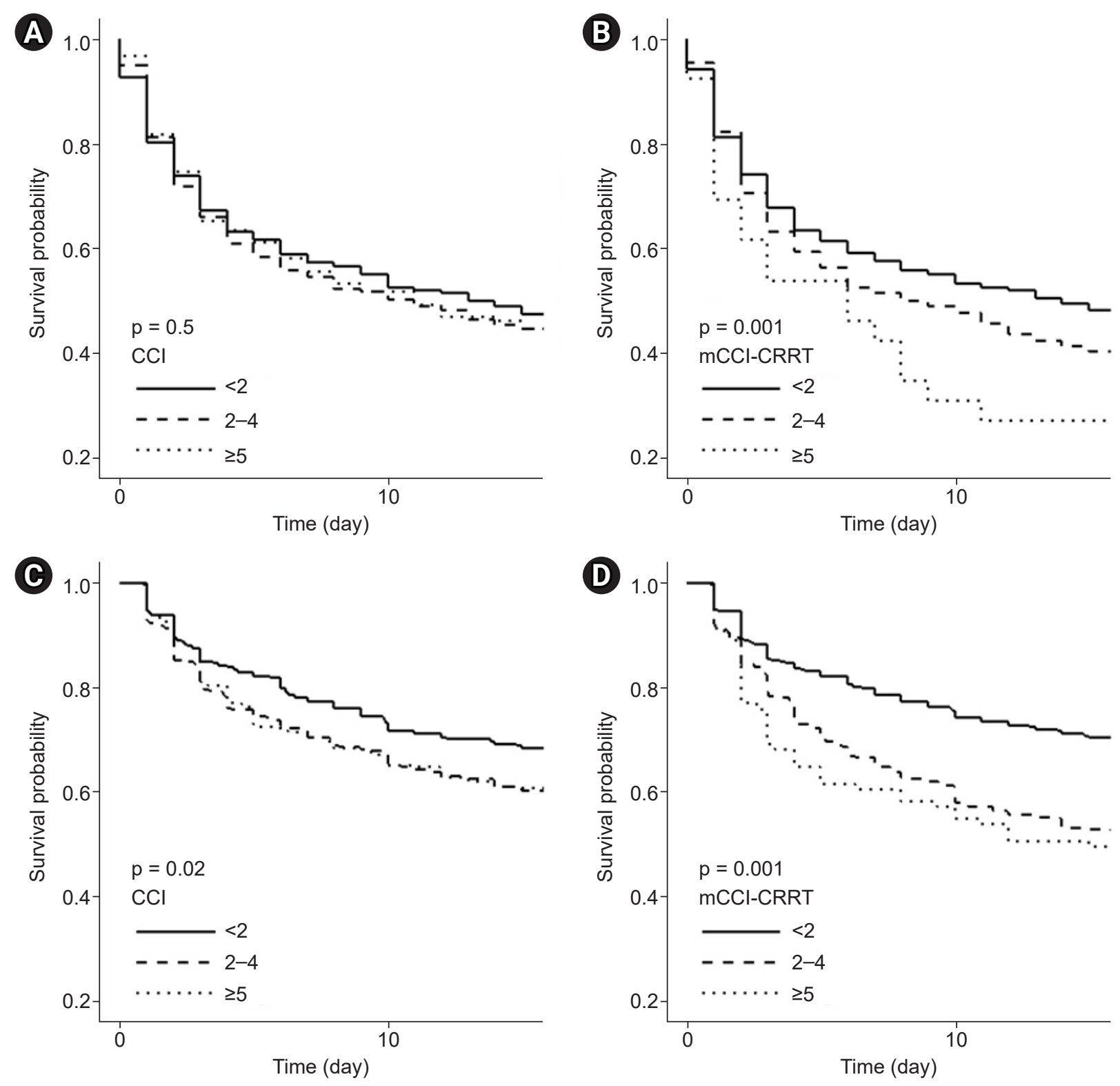

Figure 2. Kaplan-Meier curve of $\mathbf{C C I}$ and mCCI-CRRT score groups in development (A, B) and validation (C, D) cohorts.

$\mathrm{CCl}$, Charlson Comorbidity Index; mCCl-CRRT, modified CCl-continuous renal replacement therapy.

\section{Discussion}

In this study, we modified the original CCI using the AKICRRT cohort database of three hospitals in Korea to provide better risk stratification in AKI patients requiring CRRT. Furthermore, we validated the accuracy of the modified index by comparing its performance with that of the original index using data of independent hospitals for the development cohort. We found that mCCI-CRRT exhibited better risk stratification performance for mortality in incident AKICRRT patients compared with that provided by the original CCI.

In patients with severe AKI requiring CRRT, the effect of comorbidities on outcome is considerable and warrants development of a comorbidity index [12,13]. However, for the original CCI, the scoring system was created with a development cohort of patients admitted to a general ward with various disease entities, while validation was performed on 
Table 3. Model performance of the mCCl-CRRT in the validation cohort

\begin{tabular}{|c|c|c|c|}
\hline Variable & C-statistics & $\mathrm{cNRI}^{\mathrm{a}}$ & $\mathrm{cNRI}^{\mathrm{b}}$ \\
\hline \multicolumn{4}{|l|}{ CCl vs. mCCl-CRRT } \\
\hline $\mathrm{CCl}$ & $0.54(0.50-0.58)$ & $35.4(22.1-48.1)$ & $35.9(23.3-48.5)$ \\
\hline mCCl-CRRT & $0.59(0.55-0.62)$ & & \\
\hline p-value & 0.01 & 0.001 & 0.001 \\
\hline \multicolumn{4}{|c|}{ Age-adjusted CCI vs. mCCI-CRRT } \\
\hline Age-adjusted CCl & $0.58(0.54-0.61)$ & $22.9(9.9-35.8)$ & $15.2(2.1-28.2)$ \\
\hline mCCI-CRRT & $0.59(0.55-0.62)$ & & \\
\hline p-value & 0.6 & 0.001 & 0.02 \\
\hline
\end{tabular}

$\mathrm{CCl}$, Charlson Comorbidity Index; cNRI, continuous net reclassification improvement; mCCI-CRRT, modified CCl-continuous renal replacement therapy.

${ }^{a}$ Adjusted for age and sex; ${ }^{b}$ adjusted for age, sex, hemoglobin, and albumin.

breast cancer patients [4]. Therefore, it might not be appropriate to apply the original CCI in AKI patients requiring CRRT. To our knowledge, there have been few attempts to recalibrate the CCI of patients requiring CRRT. Therefore, this study is meaningful in that it used a large sample of incident AKI-CRRT patients and validated the index using an independent observational cohort.

The disease weights in the original CCI showed many changes when using the mCCI-CRRT. When considering only HR without statistical significance, the more prevalent causes of septic AKI (metastatic solid tumor, any tumor, connective tissue disease, chronic pulmonary disease, and moderate to severe liver disease) showed higher HR values. In contrast, HRs tended to be $<1.0$ in common causes of ischemic AKI, hypovolemic AKI, and postoperative AKI (peptic ulcer disease, dementia, hemiplegia, cerebrovascular disease, congestive heart failure, and myocardial infarction) [14].

Metastatic solid tumors show the highest weight, even in mCCI-CRRT [15-18]. Although target therapy and immunotherapy, which did not exist in the 1980s when the original CCI was developed, are used widely and new anticancer drugs are being developed, the mortality and risk of sepsis and septic AKI in cancer patients remain high. This highest weight also might be due to cancer treatment-related immune dysfunction and secondary infections [19,20].

Although the difference was not statistically significant, the weight of connective disease in mCCI-CRRT increased from 1 to 2 compared with the original CCI. Recently, biologics such as tumor necrosis factor- $\alpha$ or interleukin- 6 blocking monoclonal antibodies, which did not exist 25 years ago, have been used for various autoimmune diseases and connective tissue disorders [21]. The disturbance of the immune system following the use of these biologics is believed to cause sepsis more frequently and is more difficult to treat in patients with connective tissue disease [22,23]. On the other hand, the weights of diabetes and hemiplegia were lower in the mCCI-CRRT group than in the original CCI group. Since CCI was first developed in 1977, the guidelines for diabetes control have become more sophisticated, and the target serum glucose level has been lowered, which might decrease the risk of mortality due to diabetes. In this study, peptic ulcer disease showed a statistically significant hazard ratio. Previous studies have shown that the incidence of stress-induced ulcers was high in critically ill patients receiving ICU care [24-26]. In one observational retrospective study, 90-day mortality was significantly higher in a bleeding group among patients admitted to the ICU, but there was no difference in mortality at $<28$ days compared to the non-bleeding group [24]. In another study, critically important gastrointestinal (GI) bleeding was not significantly related to 90-day mortality in patients who received ICU care for more than 7 days, and 90-day mortality was increased by comorbidities such as liver disease, renal replacement therapy, and coagulopathy [27]. Finally, based on a recent randomized trial comparing proton pump inhibitor and placebo groups in critically ill patients admitted to the ICU, 90-day mortality and the number of clinically important events did not show significant difference between the two groups [28]. These studies show that GI bleeding had relatively little influence on mortality in critically ill patients compared to other comorbidities, which is consistent with the results of this study.

In both the original CCI and mCCI-CRRT, there was no 
statistically significant difference in mortality between men and women, as shown in Table 2. Previous studies have found similar lack of association. Zettersten et al. [29] reported no difference in mortality between men and women in a retrospective cohort analysis of approximately 9,000 patients admitted to a Swedish university hospital ICU between 2006 and 2016. Sakr et al. [30] reported that the overall ICU mortality rate did not differ significantly between males and females in 3,902 patients admitted to one of 24 participating medical and/or surgical ICUs between April 2006 and September 2006.

Our study has some limitations. First, risk stratification for underlying chronic diseases was performed without considering the differences in acute antecedent factors such as myocardial infarction, bleeding, or sepsis that cause AKI. In this situation, the acute medical condition requiring CRRT is severe, and the contribution of underlying chronic diseases can be underestimated. Second, this dataset only included serum hemoglobin and albumin levels among many laboratory measures. Thus, we could not adjust for other variables such as baseline serum creatinine, quantitative proteinuria, or prothrombin time for recalibrating the weights of comorbidities. In addition, since there were no patients with acquired immunodeficiency syndrome in either cohort, this condition was not taken into consideration. Third, this is a scoring system created from a cohort composed of a single racial group, and it might not be generalizable or applicable to other racial groups and populations. Additional validation using disease-specific cohorts and other national data is necessary. Fourth, since the underlying comorbidity information is based on the ICD-10 code registered in the diagnosis window of electronic health records, specific relative risk of mortality could be underestimated. Finally, since information on CRRT dose, which is an important factor for CRRT patients, was not collected, there might be a difference in CRRT dose between the development and validation cohorts. However, since the sample size of each cohort was large and the same CRRT protocol was used, a large difference in CRRT dose between the two cohorts was less likely. In addition, our study focused on pre-existing conditions, i.e., underlying comorbidities prior to CRRT initiation. Therefore, we did not consider post-CRRT factors.

A major strength of our study is that the new index was created based on a relatively large cohort from a multi-cen- ter ICU, and it was validated with an independent, similarly sized cohort, which is quite large in terms of CRRT-related studies. Lastly, the mCCI-CRRT demonstrated superior performance in a head-to-head comparison with the original CCI.

In conclusion, we suggest use of the new mCCI-CRRT scoring system that offers superior risk stratification for mortality in incident AKI-CRRT patients compared with the original CCI. In addition, this could be a preferred scoring system in clinical practice and statistical analysis in epidemiological research. As treatment options for various diseases develop, the effects of individual chronic diseases on mortality will change. Therefore, this edition of the $\mathrm{mC}$ CI-CRRT is not final, and it will require periodic updates to remain current and fit the trends over time.

\section{Conflicts of interest}

The authors declare no conflict of interest.

\section{Funding}

This research was supported by the Basic Science Research Program through the National Research Foundation of Korea (NRF), funded by the Ministry of Education (2021R1I1A3052012).

\section{Authors' contributions}

Conceptualization: JPL, JYP

Data curation: Jinwoo L, Jangwook L, JTP, CYJ, YCK

Methodology: JJ, DKK, SJS

Writing-original draft: Jinwoo L, JJ, Jangwook L

Writing-review \& editing: JTP, CYJ, SJS, JYP

All authors read and approved the final manuscript.

\section{ORCID}

Jinwoo Lee, https://orcid.org/0000-0003-0656-9364 Jiyun Jung, https://orcid.org/0000-0002-8235-0316 Jangwook Lee, https://orcid.org/0000-0003-2181-5850 Jung Tak Park, https://orcid.org/0000-0002-2325-8982 Chan-Young Jung, https://orcid.org/0000-0002-2893-9576 Yong Chul Kim, https://orcid.org/0000-0003-3215-8681 Dong Ki Kim, https://orcid.org/0000-0002-5195-7852 
Jung Pyo Lee, https://orcid.org/0000-0002-4714-1260

Sung Jun Shin, https://orcid.org/0000-0002-0777-9278

Jae Yoon Park, https://orcid.org/0000-0001-8986-7492

\section{References}

1. Chertow GM, Burdick E, Honour M, Bonventre JV, Bates DW. Acute kidney injury, mortality, length of stay, and costs in hospitalized patients. J Am Soc Nephrol 2005;16:3365-3370.

2. Uchino S, Kellum JA, Bellomo R, et al. Acute renal failure in critically ill patients: a multinational, multicenter study. JAMA 2005;294:813-818.

3. Prowle JR, Bellomo R. Continuous renal replacement therapy: recent advances and future research. Nat Rev Nephrol 2010;6: 521-529.

4. Charlson ME, Pompei P, Ales KL, MacKenzie CR. A new method of classifying prognostic comorbidity in longitudinal studies: development and validation. J Chronic Dis 1987;40:373-383.

5. Fried L, Bernardini J, Piraino B. Comparison of the Charlson Comorbidity Index and the Davies score as a predictor of outcomes in PD patients. Perit Dial Int 2003;23:568-573.

6. Athienites NV, Miskulin DC, Fernandez G, et al. Comorbidity assessment in hemodialysis and peritoneal dialysis using the index of coexistent disease. Semin Dial 2000;13:320-326.

7. Miskulin DC, Athienites NV, Yan G, et al. Comorbidity assessment using the Index of Coexistent Diseases in a multicenter clinical trial. Kidney Int 2001;60:1498-1510.

8. Cho H, Kim MH, Kim HJ, et al. Development and validation of the modified Charlson Comorbidity Index in incident peritoneal dialysis patients: a national population-based approach. Perit Dial Int 2017;37:94-102.

9. Park JY, Kim MH, Han SS, et al. Recalibration and validation of the Charlson comorbidity index in Korean incident hemodialysis patients. PLoS One 2015;10:e0127240.

10. Park JY, Kim MH, Bae EJ, et al. Comorbidities can predict mortality of kidney transplant recipients: comparison with the Charlson Comorbidity Index. Transplant Proc 2018;50:1068-1073.

11. Halligan S, Altman DG, Mallett S. Disadvantages of using the area under the receiver operating characteristic curve to assess imaging tests: a discussion and proposal for an alternative approach. Eur Radiol 2015;25:932-939.

12. Ortiz Librero M, Mon Mon C, Herrero Berrón JC, et al. The importance of comorbidity in nephrologists' assessment of acute renal failure. Nefrologia 2009;29:430-438.

13. Bagshaw SM, Laupland KB, Doig CJ, et al. Prognosis for long- term survival and renal recovery in critically ill patients with severe acute renal failure: a population-based study. Crit Care 2005;9:R700-R709.

14. Pinheiro KH, Azêdo FA, Areco KC, Laranja SM. Risk factors and mortality in patients with sepsis, septic and non septic acute kidney injury in ICU. J Bras Nefrol 2019;41:462-471.

15. Rosner MH, Perazella MA. Acute kidney injury in patients with cancer. N Engl J Med 2017;376:1770-1781.

16. Danai PA, Moss M, Mannino DM, Martin GS. The epidemiology of sepsis in patients with malignancy. Chest 2006;129:1432-1440.

17. Rosolem MM, Rabello LS, Lisboa T, et al. Critically ill patients with cancer and sepsis: clinical course and prognostic factors. $J$ Crit Care 2012;27:301-307.

18. Thirumala R, Ramaswamy M, Chawla S. Diagnosis and management of infectious complications in critically ill patients with cancer. Crit Care Clin 2010;26:59-91.

19. Wanchoo R, Karam S, Uppal NN, et al. Adverse renal effects of immune checkpoint inhibitors: a narrative review. Am J Nephrol 2017;45:160-169.

20. Shingarev R, Glezerman IG. Kidney complications of immune checkpoint inhibitors: a review. Am J Kidney Dis 2019;74:529537.

21. Rutherford AI, Subesinghe S, Hyrich KL, Galloway JB. Serious infection across biologic-treated patients with rheumatoid arthritis: results from the British Society for Rheumatology Biologics Register for Rheumatoid Arthritis. Ann Rheum Dis 2018;77:905910.

22. Vallabhaneni S, Chiller TM. Fungal infections and new biologic therapies. Curr Rheumatol Rep 2016;18:29.

23. George MD, Baker JF, Winthrop K, Curtis JR. Risk of biologics and glucocorticoids in patients with rheumatoid arthritis undergoing arthroplasty. Ann Intern Med 2019;171:680.

24. Cook DJ, Griffith LE, Walter SD, et al. The attributable mortality and length of intensive care unit stay of clinically important gastrointestinal bleeding in critically ill patients. Crit Care 2001;5:368-375.

25. Cook DJ, Fuller HD, Guyatt GH, et al. Risk factors for gastrointestinal bleeding in critically ill patients. Canadian Critical Care Trials Group. N Engl J Med 1994;330:377-381.

26. Schuster DP, Rowley H, Feinstein S, McGue MK, Zuckerman GR. Prospective evaluation of the risk of upper gastrointestinal bleeding after admission to a medical intensive care unit. $A m \mathrm{~J}$ Med 1984;76:623-630.

27. Krag M, Perner A, Wetterslev J, et al. Prevalence and outcome of gastrointestinal bleeding and use of acid suppressants in acutely 
ill adult intensive care patients. Intensive Care Med 2015;41:833845.

28. Krag M, Marker S, Perner A, et al. Pantoprazole in patients at risk for gastrointestinal bleeding in the ICU. N Engl J Med 2018; 379:2199-2208.

29. Zettersten E, Jäderling G, Bell M, Larsson E. Sex and gender aspects on intensive care: a cohort study. J Crit Care 2020;55:2227.

30. Sakr Y, Elia C, Mascia L, et al. The influence of gender on the epidemiology of and outcome from severe sepsis. Crit Care 2013;17:R50. 